\title{
Option
}

La pisciculture dans le monde

\section{Perspectives pour la recherche biotechnique en pisciculture}

\author{
Bernard Chevassus-au-Louis ${ }^{1}$ \\ Jérôme Lazard ${ }^{2}$ \\ 1 Inra \\ Laboratoire de génétique des poissons \\ 78350 Jouy-en-Josas \\ France \\ <bernard.chevassus@jouy.inra.fr> \\ ${ }^{2}$ Cirad UR "Aquaculture \\ et gestion des ressources aquatiques" \\ TA B-20/01 \\ Avenue Agropolis \\ 34398 Montpellier cedex 5 \\ France \\ <jerome.lazard@cirad.fr>
}

\begin{abstract}
Résumé
Prenant en compte le contexte international des productions halieutiques, cet article propose des orientations de recherche dans le domaine de la pisciculture. Il s'agit principalement de recherches sur la maîtrise de la qualité des milieux aquacoles, en particulier de milieux littoraux et des eaux continentales, qui intègrent la question des impacts négatifs de certaines formes d'aquaculture en vue de contribuer à préserver à la fois la qualité des produits et le potentiel de production aquacole à long terme. Des recherches pluridisciplinaires sur l'alimentation et la lutte contre les bioagresseurs, prenant en compte les spécificités des systèmes aquacoles, en particulier celles des systèmes extensifs, sont également proposées. Accompagner les initiatives visant à définir et mettre en œuvre des systèmes aquacoles répondant, aux yeux des citoyens-consommateurs, aux divers critères de la durabilité et préservant l'image favorable des produits aquatiques est également un enjeu important. Nous insistons enfin sur deux aspects liés aux relations entre les populations naturelles et les populations aquacoles et qui posent des questions de recherche originales : celui de la domestication des espèces et, à l'inverse, celui des divers impacts des individus échappés d'élevage ou délibérément relâchés.
\end{abstract}

Mots clés : développement durable ; domestication ; impact sur l'environnement ; pisciculture ; programme de recherche.

Thèmes : méthodes et outils ; pêche et aquaculture ; productions animales ; recherche scientifique.

\section{Abstract \\ Prospects for biotechnical research in fish culture}

Based on the global framework of world fisheries, this paper proposes several priorities in the field of fish culture research. Primary objectives include quality control in the aquaculture environment, pluridisciplinary research analyses on fish nutrition and feeding and bioagressors control, and research supporting initiatives to characterize and implement sustainable aquaculture actions complying with the different sustainability criteria and preserving the positive image of aquatic products. Two topics focusing on the relationships between wild and cultured populations which raise interesting research questions are also proposed: species domestication and inversely, the various impacts from individuals which have either escaped from farms or have been deliberately released.

Key words: domestication; environmental impact; fish culture; research programmes; sustainable development.

Subjects: animal productions; fishing and aquaculture; scientific and agricultural research; tools and methods. es produits aquatiques contribueront à fournir un apport important à l'alimentation mondiale (Chevassus et Lazard, 2009), mais il importe à la fois de mettre en place une gestion durable de ces ressources, trop longtemps considérées comme inépuisables, et d'accompagner le développement d'une aquaculture destinée, à échéance de 10 à 20 ans, à assurer une part majori- 
taire de la production. Nous nous proposons d'examiner dans cet article les axes de recherche qu'il convient d'encourager dans cette optique en nous concentrant sur le domaine de la pisciculture.

\section{Qualité des milieux}

Si la qualité des milieux est également importante pour le développement des élevages intensifs de crustacés et de poissons, s'y ajoute dans ce cas la question inverse de l'impact environnemental de ces élevages. Outre la question des rejets, qui sera examinée plus loin, cet impact peut être lié aux transformations du milieu suite à l'implantation d'activités aquacoles. C'est en particulier le cas du développement des productions de crevettes tropicales dans les zones de mangrove côtière, considérées comme des zones particulièrement favorables à la reproduction et à la nutrition des juvéniles de nombreuses espèces marines (cette question de l'impact des élevages de crevettes sur les mangroves fait notamment partie de la liste des 30 "points chauds " identifiés par Greenpeace parmi l'ensemble des problèmes écologiques mondiaux).

Cette critique des impacts négatifs de l'aquaculture doit à l'évidence être anticipée et intégrée dans la réflexion sur son développement, ce qui suppose de rompre avec une vision par trop positive, voire positiviste, de cette activité.

\section{Alimentation}

L'impact des rejets de l'aquaculture intensive, lié en particulier aux apports d'aliments concentrés, constitue une question majeure pour le développement de cette activité. Les recherches sur l'alimentation des systèmes aquacoles intensifs devront à la fois viser à réduire ces impacts, par une meilleure efficacité de l'aliment et de son utilisation, anticiper sur une inévitable réduction de l'utilisation des huiles et farines de poissons, en recherchant des produits végétaux de substitution (voir sur cette problématique Kaushik et Mambrini, 1995 ; Médale et al., 1998 ; Mambrini et al., 1999; Francis et al., 2001 ; Kaushik, 2004), et préserver, voire améliorer, la qualité des produits souvent considérée comme un point fort des produits aquacoles (voir par exemple l'engouement actuel pour les acides gras polyinsaturés).

Deux remarques sont à faire à ce sujet. La première est que ces impératifs peuvent se révéler antagonistes, du fait notamment de la présence de facteurs antinutritionnels dans les extraits protéiques végétaux, d'une moindre appétence de ces produits, conduisant à des pertes d'aliments, ou d'une composition moins favorable des huiles végétales. La seconde est que le nécessaire désengagement de l'utilisation des farines et huiles de poissons ne tient pas qu'à des considérations économiques et pourra être hâté par l'attitude des consommateurs dans certains pays telle que le refus de "détourner " de l'alimentation humaine directe une ressource devenant rare ou la perception d'un risque sanitaire possible, à l'image du prion pour les ruminants. Dans ce dernier cas, même s'il est sans doute limité à ce groupe, le franchissement de la "barrière d'espèce " est un phénomène connu chez les poissons. Il est par exemple vraisemblable qu'une virose de la truite arcen-ciel, la septicémie hémorragique virale (SHV), soit venue de l'adaptation d'un virus des clupéidés, du fait de l'alimentation en harengs frais pratiquée notamment au Danemark avant la généralisation des aliments granulés (Dixon, 1999; Einer-Jensen et al., 2004).

Cette recherche d'une meilleure alimentation des élevages intensifs devra donc intégrer l'ensemble de ces questions et se faire de manière pluridisciplinaire, en mobilisant à la fois les sciences biologiques (nutrition, éthologie, génétique, écologie), technologiques (fabrication des aliments, systèmes d'alimentation et de traitement des effluents) et sociales (perception des consommateurs).

\section{Lutte contre les bioagresseurs}

Un autre aspect important de l'aquaculture, en particulier intensive, est celui de la lutte contre les bioagresseurs (virus, bactéries, parasites, etc.). La question se pose en effet de manière sensiblement différente de celle des élevages terrestres: un isolement sanitaire strict des sites aquacoles est le plus souvent difficile à garantir ; la panoplie thérapeutique est plus restreinte et pose souvent des problèmes environnementaux (impact des rejets, en particulier d'antibiotiques) ou de qualité sanitaire des produits (résidus); la protection vaccinale se heurte, d'une part, à une moindre connaissance - et donc une moindre maîtrise - de la réponse immune des poissons et a fortiori des invertébrés et, d'autre part, à une diversité d'espèces rendant peu rentable le développement de vaccins spécifiques. En outre, la mise en place de traitements est souvent techniquement difficile, par exemple dans des cages en mer, et leur rentabilité économique incertaine.

C'est pourquoi les stratégies de lutte devront plutôt s'inspirer de celles utilisées dans les espèces végétales avec, en particulier, l'utilisation de résistances génétiques obtenues par diverses méthodes (sélection, hybridation, voire transgenèse), l'identification et l'induction de mécanismes de défense non spécifiques, ces outils étant combinés à des approches d'écopathologie visant à comprendre, et éventuellement moduler, les conditions environnementales et les pratiques d'élevage favorisant l'émergence d'une situation pathogène. Comme dans le cas de l'alimentation, on perçoit donc ici la nécessité d'une approche systémique et pluridisciplinaire de cette question.

\section{Systèmes durables de production aquacole}

Outre le fait que leur viabilité économique soit assurée et leurs impacts environnementaux maîtrisés, le fait que les systèmes de production aquacoles soient considérés par les citoyens-consommateurs comme adaptés à leurs attentes et à leurs besoins jouera un rôle déterminant dans l'avenir, dans un contexte de forte substituabilité de la consommation des différentes productions animales, au moins dans les pays développés. Même si les prix relatifs demeurent un déterminant puissant, on observe en effet que les facteurs de compétitivité "hors prix " jouent désormais un rôle important dans les comportements d'achat de produits alimentaires. Ces facteurs peuvent concerner les aspects liés aux conditions de travail ou de rémunération des producteurs (sur le modèle du commerce équitable), aux modes d'élevage (bienêtre animal, utilisation d'antibiotiques ou 
d'OGM dans l'alimentation, etc.), au type d'animaux élevés (espèces locales ou introduites, animaux "génétiquement modifiés"), à l'impact environnemental des élevages.

Des initiatives intégrant ces aspects ont été prises dans ce sens dans le domaine des pêcheries, comme le "Code de conduite pour une pêche responsable ", adopté en 1995 par la FAO, ou, pour l'aquaculture, le même type de code de conduite adopté en 1997, le développement de l'aquaculture biologique (l'aquaculture biologique représenterait actuellement en Europe environ 14000 tonnes de production, dont 11000 tonnes de saumons (Lane, 2006), soit environ $1 \%$ de la production de poissons ; la conchyliculture n'utilise pas cette référence, même si son mode de production peut être considéré comme totalement " biologique "), de divers signes de qualité (Label Rouge, norme Afnor) ou la Global Aquaculture Alliance (www.gaalliance.org), fondée par des producteurs de crevettes pour répondre aux critiques sur l'impact environnemental de ces élevages et proposer un code de bonnes pratiques.

Au nom de l'équité sociale et notamment dans les PED, les organismes et institutions en charge de la pisciculture s'attacheront chaque fois que possible, notamment au travers de politiques publiques adaptées, à faire de cette activité un "outil de développement ".

La recherche devra donc se mobiliser pour conforter ces démarches, en définissant des itinéraires techniques adaptés et en fournissant des critères pertinents d'évaluation de leur efficacité et de leur durabilité.

\section{Aquaculture extensive : problématiques originales}

Il apparaît (Chevassus et Lazard, 2009) que la production en eau douce de poissons de faible valeur est, et demeurera, une composante majeure de l'aquaculture. Cette production est basée sur une gestion "agronomique " des plans d'eau (c'est pourquoi on la qualifie parfois "d'aquaculture de production", à l'opposé de "l'aquaculture de transformation" basée sur l'apport d'aliments concentrés), à savoir la stimulation de la productivité naturelle par divers fertilisants minéraux, et surtout organiques (fumiers, lisiers, fientes de volailles, débris végétaux), une partie de ces derniers pouvant d'ailleurs être consommés directement par les poissons. On peut supposer que, pour cette activité issue d'une longue tradition - le premier " traité de pisciculture " connu est celui de Fan Li, écrit en Chine en 473 av J.-C. (Billard, 2000) - les pratiques empiriques ont pratiquement optimisé le système de production, comme le montrent par exemple les analyses de Dey et al. (2000) et de Irz et McKensie (2003) sur les inefficacités techniques de la production de tilapias en Asie du Sud-Est. En revanche, ces systèmes sont confrontés à une nécessaire intensification, les surfaces dévolues à l'aquaculture ne semblant pas pouvoir s'étendre notablement dans la plupart des pays concernés.

Parmi les facteurs d'intensification envisagés, le recours à l'apport complémentaire d'aliments concentrés, du type de ceux utilisés en aquaculture intensive, constitue une piste tentante et déjà mise en œuvre dans de nombreux cas, là où les conditions économiques rendaient cette option attrayante (Hepher, 1988). C'est par exemple le cas pour le développement récent de la production de tilapias en Égypte (FAO, 2003) et de carpes en Chine (Li, 2002). Cependant, outre les limites prévisibles de l'utilisation des farines et huiles de poissons, il apparaît que l'utilisation d'aliments concentrés dans de tels systèmes aquacoles contribue davantage à stimuler la productivité naturelle de l'étang en algues ou invertébrés qu'à nourrir directement les poissons, d'où une très faible efficacité économique (Schroeder, 1983). Tacon (1997), pour sa part, évalue à $30 \%$ au minimum le surnourrissage des élevages et la surformulation en protéines des aliments utilisés en aquaculture tout particulièrement en élevage semi-intensif en étangs.

L'intensification raisonnée des systèmes extensifs est donc un enjeu complexe, qui devra combiner les savoirs traditionnels et les démarches scientifiques d'écologues, de nutritionnistes, de physicochimistes, etc. Cette recherche devra également tenir compte de l'intégration de ces systèmes aquacoles dans les territoires ruraux et la diversité de leurs activités, tant en termes de contraintes (gestion de l'eau, pluriactivité éventuelle des aquaculteurs entre autres) que d'opportunités et de complémentarités (utilisation de déchets de l'exploitation, de sous- produits agricoles ou, à l'inverse, utilisation des effluents aquacoles par l'agriculture).

Un autre aspect spécifique de l'aquaculture extensive est le recours fréquent à la polyculture, les différentes espèces élevées (poissons, mais aussi crustacés d'eau douce) jouant des rôles écologiques différents et complémentaires. Cet aspect doit être pris en compte dans la problématique précédemment évoquée de l'alimentation mais pose également des problèmes intéressants et originaux pour l'amélioration génétique : l'amélioration d'une seule espèce, par exemple, en termes de vitesse de croissance ou de comportement alimentaire, peut en effet se traduire par une production plus importante de cette espèce au détriment des autres, la production globale du milieu n'étant pas améliorée. La question de l'amélioration des performances d'un peuplement multispécifique sous contrainte trophique globale (la productivité totale potentielle du milieu) est donc beaucoup plus complexe que celle de l'amélioration d'une production monospécifique non limitée par l'apport d'intrants. En effet, dans ce dernier cas, l'amélioration de la croissance par sélection semble résulter essentiellement d'une augmentation de la consommation journalière spontanée, sans amélioration de l'efficacité des aliments, comme cela a été montré chez les salmonidés (Chevassus et al., 2004 ; Mambrini et al., 2004a et 2004b).

\section{Domestication}

Est principalement intégrée dans cette rubrique la question du bouclage en captivité du cycle biologique des espèces avec, en particulier, la maitrise de la maturation sexuelle et la production abondante et régulière de juvéniles à des coûts économiquement acceptables. Une question connexe que nous discuterons est celle de la constitution et de la gestion des ressources génétiques des stocks domestiques, dès lors que le recours à des prélèvements dans le milieu naturel deviendra exceptionnel. Du fait de la diversité des espèces déjà élevées avec succès, on pourrait considérer que les recherches dans ce domaine ne constituent pas une priorité. Plusieurs faits militent cependant pour les encourager. 
Le premier est qu'une partie importante de l'aquaculture - environ $20 \%$ selon la FAO (SOFIA, 2004) - est basée sur le prélèvement de juvéniles, voire de subadultes, dans le milieu naturel. Les exemples de l'anguille, de la sériole japonaise, d'une grande partie de la production de mollusques et, plus récemment, des élevages de thons sont connus, et ces prélèvements, lorsqu'ils deviennent importants, peuvent être parfois considérés comme dommageables pour les populations naturelles. Ils peuvent également se révéler un facteur limitant du développement aquacole, du fait de leur caractère parfois limité dans le temps, de leur fluctuation d'abondance, de l'hétérogénéité des performances selon les lots ou des risques sanitaires liés à ces introductions. Ainsi, la maitrise de la production de juvéniles de poissons-chats du Mékong (genre Pangasius) a été l'une des clés majeures du développement de ces élevages, dont la production est passée de 50000 tonnes en 1996 à 1000000 tonnes estimés en 2007 (Cacot et Lazard, 2004 ; Lazard et al., 2007). Enfin, la mâtrise du cycle biologique est une condition indispensable à la mise en place de programmes d'amélioration génétique, dont l'efficacité est maintenant bien établie pour plusieurs espèces de poissons.

La seconde considération est qu'une partie également importante de l'aquaculture est basée sur un petit nombre d'espèces, qui ont été largement disséminées à travers le monde : les tilapias, d'origine africaine mais largement répandus à travers l'Asie (Lazard, 1993), l'huître japonaise, à l'origine de l'essentiel de la production européenne, ou le saumon atlantique, maintenant élevé aussi bien au Chili qu'en Alaska, en sont des exemples. Si certaines espèces d'introduction ancienne (comme la carpe commune ou la truite arc-en-ciel en France) sont maintenant naturalisées et, à ce titre, considérées comme indigènes, d'autres suscitent des critiques et des inquiétudes légitimes sur l'impact éventuel - direct ou indirect (introduction conjointe d'agents pathogènes) - de leur introduction (Lazard, 1994). C'est notamment le cas pour l'introduction de tilapias dans les milieux insulaires (Hostache, 1998 ; Anonyme, 2004) ou de crevettes du Pacifique en Inde (Raghavan et Prasad, 2006). Développer une compétence sur la domestication d'espèces indigènes apparaît donc nécessaire pour anticiper une vraisemblable restriction des introductions d'espèces et permettre le développement aquacole dans des pays où cette activité est encore très limitée (Afrique, Amérique du Sud).

Enfin, la question de la mise en place d'une véritable gestion dynamique des ressources génétiques des populations d'élevage - qui inclut à la fois l'inventaire et la collecte de la diversité naturelle et la définition de pratiques permettant la conservation de cette diversité dans des populations fermées - est une problématique qui concerne également des espèces pour lesquelles la domestication a été réalisée, parfois depuis de longues années. On observe, en effet, souvent que les populations d'aquaculture n'ont incorporé ou n'ont conservé qu'une fraction de la diversité génétique des populations naturelles (Guyomard, 1989, pour la truite commune ; Eknath et al., 1993, pour le tilapia du Nil) et que le rééchantillonnage de cette diversité - lorsqu'il est encore possible - peut conduire à des gains substantiels de performance (Wohlfarth et al., 1975, chez la carpe commune ; Chevassus et al., 1992, chez la truite commune ; Bentsen et al., 1998, chez le tilapia du Nil).

Comprendre et maitriser la dynamique évolutive des populations fermées, en particulier les rôles respectifs de la dérive génétique (lié à un nombre souvent faible de reproducteurs), de la sélection naturelle (par viabilité ou mortalité différentielle) et des sélections plus ou moins intentionnelles pratiquées par les éleveurs et analyser les interactions entre ces différents processus apparaît donc un enjeu global pour l'aquaculture (Vandeputte et Launey, 2004). Les outils et concepts de la génétique et de la génomique des populations, de l'écologie et de l'éthologie seront à mobiliser pour ces recherches.

\section{Impact \\ sur les populations naturelles}

La plupart des populations d'aquaculture continuent à avoir des liens étroits avec les populations naturelles. Il peut s'agir, comme nous l'avons vu, de prélèvement de juvéniles ou de reproducteurs dans le milieu naturel pour alimenter les élevages ou, à l'inverse, d'introductions volontaires (repeuplements) ou fortuits (échappement des enceintes d'élevage). Un cas intermédiaire est le relâcher délibéré d'animaux que les aquaculteurs jugent "peu performants " et ne souhaitent pas conserver en élevage. Le gouvernement de l'Alaska estime par exemple que cette pratique concerne 3 à $5 \%$ des effectifs des élevages de saumon atlantique (Anonyme, 2002). Pour ne donner qu'un exemple, le gouvernement norvégien publie dans ses statistiques officielles le nombre de saumons échappés chaque année des élevages (www.ssb.no/en/fiskeoppdrett). En 2004, ce nombre s'est élevé à 348 600, alors que la biomasse en cage était de 356000 tonnes, soit environ 200 millions d'individus. Le taux d'échappement serait donc très inférieur à $1 \%$, mais l'effectif de cette population échappée serait supérieur à celui du total des adultes sauvages rentrant dans les rivières de ce pays, estimé à environ 200000 individus (Heuch et Mo, 2001), d'où de forts impacts possibles sur ces populations naturelles.

Cette situation, assez originale par rapport à la plupart des élevages terrestres, oblige donc à s'interroger sur les conséquences éventuelles de ces introductions. Plusieurs types d'impacts peuvent être envisagés :

- démographiques, lorsque ces échanges peuvent avoir des conséquences notables sur l'abondance des populations naturelles. Outre le cas évident des prélèvements, il peut s'agir, pour des individus échappés ou relâchés, de compétition - favorable aux animaux d'aquaculture, par exemple du fait d'une taille plus élevée - pour une ressource limitante (territoire, alimentation, accès aux femelles pour la reproduction, etc.), aboutissant à un "déplacement" des populations naturelles. Ce phénomène peut d'ailleurs advenir, lorsque les relâchers sont importants, sans que ceux-ci aient un avantage particulier, par simple effet de "dilution ";

- sanitaires, lorsque les animaux d'aquaculture sont porteurs d'agents pathogènes s'étant multipliés en élevage et transmissibles aux populations naturelles ;

- génétiques, lorsque les animaux introduits sont porteurs d'un patrimoine génétique dont l'introgression totale ou partielle dans les populations naturelles peut affecter, à plus ou moins long terme, leur valeur sélective. On peut considérer qu'il s'agit dans ce cas d'un impact démographique "différé ".

Cette typologie des impacts est à croiser avec une autre, basée sur le degré de similitude génétique entre les populations d'élevage et les populations naturelles et sur les possibilités de reproduction croi- 
Tableau 1. Typologie différents impacts possibles des populations d'aquaculture sur les populations naturelles.

Table 1. Typology of the different potential impacts from aquacultural populations on natural populations.

\begin{tabular}{|c|c|c|c|}
\hline \multirow[t]{2}{*}{ Impacts } & \multicolumn{3}{|c|}{ Différenciation } \\
\hline & Génétiquement proches et interfertiles & $\begin{array}{l}\text { Génétiquement différenciées mais } \\
\text { sexuellement " compatibles » }\end{array}$ & Non interfertiles \\
\hline Démographiques & \multicolumn{2}{|c|}{ Faibles, sauf si flux importants et récurrents } & $\begin{array}{l}\text { Potentiellement } \\
\text { forts si espèce } \\
\text { invasive }\end{array}$ \\
\hline Sanitaires & \multicolumn{3}{|c|}{ Potentiellement importants } \\
\hline Génétiques & $\begin{array}{l}\text { Néant, sauf animaux sexuellement } \\
\text { inversés avec flux importants (perturbation } \\
\text { du sex-ratio de la population) }\end{array}$ & $\begin{array}{l}\text { Variables et mal documentés; en théorie, } \\
\text { potentiellement forts dans certains cas }\end{array}$ & Néant \\
\hline
\end{tabular}

sée entre ces deux ensembles. On peut là aussi distinguer trois niveaux dans ce continuum :

- des populations fertiles et génétiquement peu différenciées par rapport aux populations naturelles (y compris des populations sexuellement inversées par voie hormonale) ;

- des populations ayant un génotype nettement différent des populations naturelles mais dont les individus peuvent néanmoins s'intégrer dans le pool de reproducteurs de ces populations. Cette catégorie regroupe à la fois des populations d'aquaculture issues d'autres populations naturelles, des populations dont la différenciation est due à un programme d'amélioration génétique ou à diverses modifications du système de reproduction (hybrides interspécifiques fertiles, polyploïdes, animaux transgéniques, etc.), ou des espèces proches et interfécondes. Il convient de distinguer dans ce vaste ensemble deux extrêmes : le cas de croisements avec les populations naturelles donnant des individus parfaitement viables et fertiles et, à l'inverse, le cas de croisements aboutissant à des produits inviables ou viables et stériles, avec tous les intermédiaires possibles en termes de réduction de viabilité ou de fertilité ;

- des populations non susceptibles de participer à la reproduction des populations naturelles : espèces éloignées, animaux totalement stériles.

Le croisement de ces deux typologies permet de préciser les différents risques à considérer (tableau 1) : les impacts possibles de populations peu différenciées sont principalement sanitaires, ceux de populations sexuellement incompatibles, démographiques et sanitaires, ceux enfin de populations différenciées mais sexuellement compatibles pouvant être multiples. La littérature sur les espèces aquatiques est riche de considérations théoriques ou d'observations empiriques permettant d'illustrer ces différentes situations, et il serait trop long de la présenter ici. Nous soulignerons en revanche que, une fois admise l'éventualité de tels impacts, la capacité de les évaluer et surtout de les quantifier a priori, dans une situation concrète donnée, nous semble limitée, alors que cette expertise deviendra de plus en plus nécessaire pour le développement de l'aquaculture. Le Protocole de Carthagène (2000) sur les mouvements transfrontières d'OGM illustre bien cet impératif, et de récents débats autour des impacts éventuels d'huîtres tétraploïdes (voir par exemple le site www.terresacree.org/huitre.htm) ou de la réintroduction en Afrique de tilapias sélectionnés en Asie pour la croissance soulignent également la nécessité de renforcer les recherches et la capacité d'expertise dans ce domaine.

\section{Conclusion}

Les questions évoquées dans cet article concernent certaines des recherches qui paraissent devoir être menées en priorité et illustrent une des problématiques majeures du développement durable, celle de la solidarité intragénérationnelle. Elle concerne en effet la contribution des pays développés, aujourd'hui détenteurs de la majorité des compétences de recher- che, à la définition et à la mise en œuvre du développement de l'aquaculture, enjeu qui concerne en priorité les pays en développement. Si les pays émergents majeurs, comme la Chine, l'Inde et le Brésil sont appelés à devenir à court terme " autosuffisants " en termes de recherche, voire exportateurs de compétences, il n'en est pas de même pour de nombreux autres pays fortement concernés par ces ressources. Le fait que les pays développés - et leur dispositif de recherche - se focalisent essentiellement sur la gestion de leurs propres ressources ou prennent en compte des enjeux plus globaux sera donc un test significatif de leur volonté de contribuer effectivement au développement durable de la planète.

\section{Références}

Anonyme. Atlantic Salmon: a white paper. 13 pp. Alaska Department of Fish and Game (site: www.adfg.state.ak.us:special/as/docs/ as_white2002.pdf), 2002.

Anonyme. Une action drastique dans un paradis tropical. GISPNEWS, Bulletin du Global Invasive Species Programme, 2, 16. (site: www.gisp.org) 2004.

Bentsen $\mathrm{HB}$, Eknath $\mathrm{AE}$, Palada-de-Vera MS, et al. Genetic improvement of farmed tilapias: growth performance in a complete diallel cross experiment with eight strains of Oreochromis niloticus. Aquaculture 1998; 160 : 145-73.

Billard R. Le traité de Fan Li ( $5^{\mathrm{e}}$ siècle av. J.-C.) et la pisciculture en Chine. II Pesce 2000; (février) : 54-61.

Cacot P, Lazard J. Domestication d'espèces de poissons-chats du Mékong de la famille des Pangasiidae. Inra Prod Anim 2004 ; 17 : 195-8.

Chevassus B, Krieg F, Guyomard R, Blanc JM, Quillet E. The genetics of brown trout: twenty years of French research. Buvisindi Icel Agri Sci $1992 ; 6: 109-24$ 
Chevassus B, Quillet E, Krieg F, et al. Enhanced individual selection for selecting fast growing fish: the "PROSPER " method with application on brown trout (Salmo trutta fario). Genet $\mathrm{Se}$ Evol 2004 ; 36 : 643-61.

Chevassus B, Lazard J. Situation et perspectives de la pisciculture dans le monde : consommation et production. Cah Agric 2009; 18: 82-90. doi : 10.1684 /agr.2009.0283

Dey MM, Paraguas FJ, Bimbao GB, Regaspi PB. Technical efficiency of tilapia growout pond operations in the Philippines. Aqua Eco Management $2000 ; 4: 33-48$.

Dixon PF. VHSV came from the marine environment: clues from the literature or just red herrings? Bull Eur Ass Fish Pathol 1999: 19: 60-5.

Einer-Jensen $\mathrm{K}$, Ahrens $\mathrm{P}$, Forsberg $\mathrm{R}$, Lorenzen N. Evolution of the fish rhabdovirus viral haemorrhagic septicemia virus. JGen Virol $2004 ; 85$ : 1167-79.

Eknath AE, Tayamen MM, Palada-de-Vera MS et al. Genetic improvement of farmed tilapias: the growth performance of eight strains of Oreochromis niloticus tested in different farm environments. Aquaculture 1993; 111: 171-88.

FAO. État de l'aquaculture dans le monde. Rome: FAO, 2003.

Francis G, Makkar HPS, Becker K. Antinutritional factors present in plant-derived alternate fish feed ingredients and their effects in fish. Aquaculture 2001 ; 199 : 197-227.

Guyomard R. Diversité génétique de la truite commune. Bull Fr Pêche Piscic 1989; 314 : 118-35.

Hepher B. Nutrition of pond fishes. Melbourne (Australie) : Cambridge University Press, 1988.

Heuch MA, Mo TA. A model of salmon louse production in Norway: effects of increasing salmon production and public management measures. Dis Aquat Organ 2001 ; 45 : 145-52.
Hostache G. Réflexions sur la gestion des eaux douces dans le cadre de l'élaboration du schéma directeur d'aménagement du parc national de Guadeloupe: préserver le bas... pour conserver le haut. Le Courrier de I'Environnement 1998; 34: 115-118 (site: www.inra. $\mathrm{fr} / \mathrm{dpenv} /$ )

Irz X, McKensie V. Profitability and technical efficiency of aquaculture systems in Pampanga, Philippines. Aquac Econ Manag 2003; $7: 195-212$

Kaushik S. Alimentation humaine, ressources halieutiques et avenir de I'aquaculture. In: Boujard T. (dir.) Aquaculture et environnement: 20-25. Les dossiers de l'environnement de I'Inra $n^{\circ} 26$. Paris: Inra, 2004. (www.inra.fr/ dpenv/).

Kaushik S, Mambrini M. Nutrition azotée des poissons: remplacement partiel ou total de la farine de poissons. Pisc Fr 1995 ; 118: 12-20.

Lane A. The European Commission addresses organic aquaculture. World Aquac 2006; 37 : 16-8.

Lazard J. Réflexions sur la recherche en aquaculture tropicale : le tilapia d'Afrique et le tilapia d'Asie. La Jaune et la Rouge (Revue des anciens élèves de l'École Polytechnique) 1993 ; 489 : 23-6.

Lazard J. Introductions et transferts d'espèces en pisciculture. Nécessité ou opportunisme? Rev Elev Med Vet Pays Trop 1994 ; 47 : 435-8.

Lazard J, Hung LT, Cacot P. La filière "panga" (poisson-chat du Mékong) au Vietnam: les (poisson-chat du Mékong) au Vietnam: les
déterminants d'une (r)évolution aquacole. Premières journées "Recherche Filière Piscicole ", Paris, 3-4 juillet 2007 (cédérom, sous presse).

Li SF. Aquaculture research and its relation to development in China. In : Zhang LX, Liu J, Li SF, Yang NS, Gardiner PR, eds. Agricultural development and the opportunities for aquatic resources research in China. Penang (Malaysia): WorldFish Center, 2002.
Mambrini $M$, Roem AJ, Cravedi JP, Lallès JP, Kaushik SJ. Effects of replacing fish meal with soy protein concentrate and of DL-methionine supplementation in high-energy, extruded diets on the growth and nutrient utilization of rainbow trout, Oncorhynchus mykiss. J Anim Sci $1999 ; 77: 2990-9$.

Mambrini M, Médale F, Sanchez MP, et al. Selection for growth in brown trout increases feed intake capacity without affecting maintenance and growth requirements. J Anim Sci $2004 ; 82: 2865-75$

Mambrini M, Sanchez MP, Chevassus B, Labbé $L$, Quillet $E$, Boujard T. Selection for growth increases feed intake and affects feeding behaviour of brown trout. Livest Prod Sci 2004 ; 88 : 85-98.

Médale $\mathrm{F}$, Boujard $\mathrm{T}$, Vallée $\mathrm{F}$, et al. Voluntary feed intake nitrogen and phosphorus losses in rainbow trout (Oncorhynchus mykiss) fed increasing dietary levels of soy protein concentrate. Aquat Living Resour 1998; 11 : 239-46.

Raghavan R, Prasad G. A needless diversification: a perspective on the unregulated introduction and culture of the Pacific white shrimp, Litopenaeus vannamei, in India. World Aquac 2006 ; 37 : 8-12.

Schroeder GL. Sources of fish and prawn growth in polyculture ponds as indicated by ¿C analysis. Aquaculture $1983 ; 35: 29-42$.

SOFIA. The state of world fisheries and aquaculture. Rome: FAO, 2004.

Tacon AGC. Selected developments and trends/Aquafeeds and feeding strategies. In FAO, ed. Review of the state of world aquaculture. FAO Fisheries Circular $\mathrm{n}^{\circ}$ 886, Rev. 1 Rome (Italie): FAO, 1997.

Vandeputte M, Launey S. Quelle gestion génétique de la domestication chez les poissons? Inra Prod Anim 2004 ; 17 : 237-42.

Wohlfarth GW, Moav R, Hulata G. Genetic differences between the Chinese and European races of the common carp. II. Multicharacter variation $-a$ response to the diverse methods of fish cultivation in Europe or China. Heredity $1975 ; 34: 341-50$. 